\title{
Presence of $\alpha$ smooth muscle actin in lens epithelial cells of aphakic rabbit eyes
}

\author{
Daijiro Kurosaka, Katsuhiko Kato, Toshiyuki Nagamoto
}

\begin{abstract}
Aims-To determine whether $\alpha$ smooth muscle actin ( $\alpha-S M A)$, a marker for myofibroblastic cells, is present in lens epithelial cells (LECs) in rabbit aphakic eyes.

Methods-Phacoemulsification was performed in rabbit eyes, which were enucleated after surgery. Immunohistochemical methods were used to detect $\alpha-S M A$ in LECs.

Results-Five days after surgery, the presence of $\alpha$-SMA positive LECs was observed mainly around the adhesive portion of the anterior capsule margin and the posterior capsule. The posterior capsule was wrinkled at the adhesive portion. The $\alpha$-SMA positive LECs were flattened with spindle-shaped cross sections. Seven days after surgery, the $\alpha$-SMA positive LECs covered most of the central posterior capsule. They disappeared 10 days after surgery. On the other hand, the cuboidal LECs in the capsular bag were negative for $\alpha$-SMA.

Conclusion-The flattened LECs with spindle-shaped cross sections observed 5 days after cataract surgery contained $\alpha-S M A$. Such LECs were distinguished biochemically from the cuboidal LECs, which lacked $\alpha$-SMA.
\end{abstract}

(Br F Ophthalmol 1996;80:906-910)

Department of

Ophthalmology, Keio

University School of

Medicine, Tokyo, Japan

D Kurosaka

K Kato

Department of Developmental Biology, National

Institute for Basic

Biology, Okazaki,

Japan

T Nagamoto

Correspondence to: Daijiro Kurosaka, $\mathrm{MD}$, Department of

Ophthalmology, Keio

University School of

Medicine, 35-Shinanomachi,

Shinjuku-ku, Tokyo, 160

Japan.

Accepted for publication 28 June 1996 of ${ }^{14}$ that is involved in the contractity fibroblasts depends on $\alpha$-SMA expression and is inhibited by the microinjection of an $\alpha$-SMA antibody into these cells. ${ }^{15}$ The ability of retinal pigment epithelial cells to exert tractional
Following cataract surgery, the residual lens proliferate. ${ }^{1-6}$ Owing to their contractile ability, cation and contraction of the lens capsule ${ }^{4-12}$ This directly causes visual disturbances ${ }^{4}$ such as a narrowing of the anterior capsular of an ${ }^{8-10}$ retinal detachment, ${ }^{11}$ decentration hypotony. ${ }^{12}$

Alpha smooth muscle actin ( $\alpha-S M A)$ is an isoform of actin and a marker for myoid differ- forces on their matrix increases with continued passage in vitro, and is correlated with the de novo expression of $\alpha$-SMA. ${ }^{16}$ We recently reported that the contractility of LECs induced by growth factors also depends on the expression of $\alpha$-SMA in vitro. ${ }^{17}$

The LECs from anterior subcapsular cataract (ASC), but not those from the normal lens, express $\alpha$-SMA in vivo. ${ }^{18}$ The LECs involved in ASC are flattened rather than cuboidal, and are surrounded by collagen fibrils. ${ }^{1920}$ They cause the lens capsule to contract. ${ }^{190}$ Following cataract surgery, the LECs that produce capsular contraction become flattened and are surrounded by collagen fibrils. ${ }^{4-7}$ Ultrastructurally, these post-cataract surgery LECs resemble those of ASC. ${ }^{7}$ These observations suggested that the flattened LECs found after cataract surgery also express $\alpha-S M A$ in vivo. The presence of $\alpha$-SMA has been reported only in the intercellular stroma of the LECs observed in the fibrotic membrane removed from the surface of the optic of the intraocular lens of the patient. ${ }^{21}$ We conducted the immunohistochemical study in aphakic rabbit eyes to better understand the relation between $\alpha$-SMA and the LECs after cataract surgery.

\section{Material and methods}

A total of 48 young Japan albino rabbits, weight $1.0-1.5 \mathrm{~kg}$, were studied. Each of the following agents was applied topically three times on the day of surgery to one eye of each rabbit: diclofenac sodium, tropicamide, phenylephrine hydrochloride, and nofloxacin. Animals were anaesthetised with intravenous pentobarbitone sodium ( $40 \mathrm{mg} / \mathrm{kg}$ ) and atropine sulphate $(0.02 \mathrm{mg} / \mathrm{kg})$. A superior corneal incision was made with a $3 \mathrm{~mm}$ keratome, and a viscoelastic material (hyaluronic acid, Pharmacia, Uppsala, Sweden) was injected into the anterior chamber. Continuous circular capsulorhexis of the anterior capsule was carried out using a capsulorhexis forceps. The lens nucleus was emulsified and the residual cortex was removed with a phacoemulsifier (Cavitron 9001, Alcon Surgical, Fort Worth, TX, USA). The corneal incision was closed with a continuous 10-0 nylon suture. At the end of the operation, gentamicin $(10 \mathrm{mg})$ was injected subconjunctivally and an antibiotic ointment (oxytetracycline hydrochloride) was applied to 


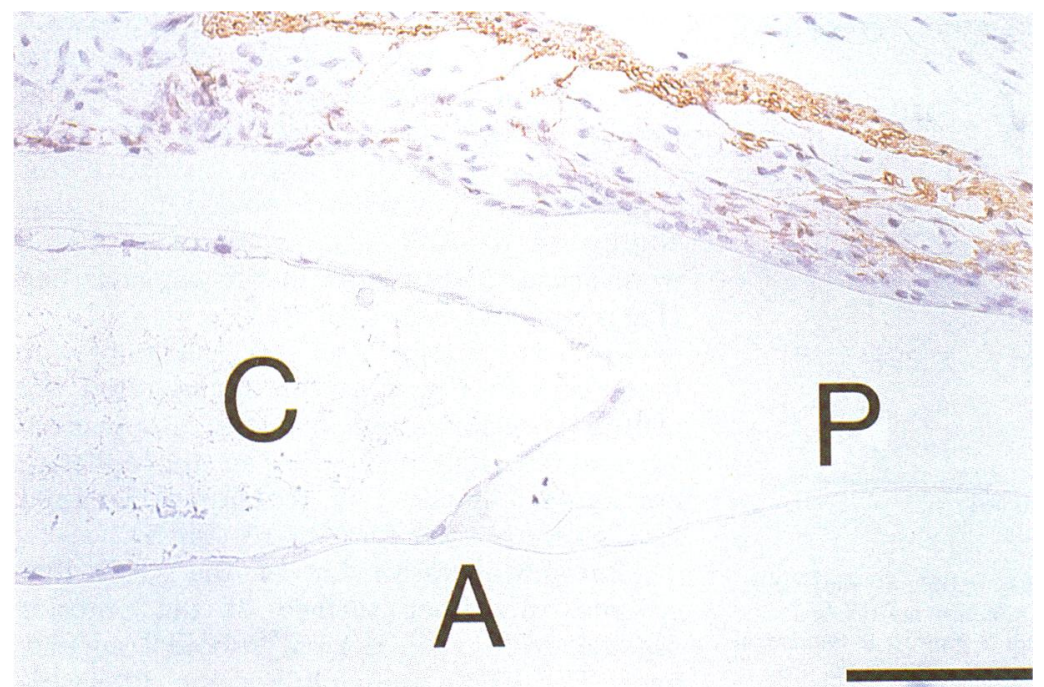

Figure 1 Immunolocalisation of $\alpha$ smooth muscle actin ( $\alpha-S M A)$ in aphakic rabbit eyes 1 day after cataract surgery. Adhesion of the anterior capsule margin to the posterior capsule divided the postoperative lens into three portions: the central posterior capsule $(P)$, the adhesive portion $(A)$, and the capsular bag $(C)$. In the capsular bag, flattened lens epithelial cells (LECs) migrate to the posterior capsule via the adhesive portion. However, they do not migrate to the central posterior capsule. These LECs are negative for $\alpha-S M A$, although the sphincter and dilator muscles of the iris are positive for $\alpha-S M A$. Sections were stained with 3-3'-diaminobenzidine hydrochloride and counterstained with haematoxylin. Bar $=100 \mu \mathrm{m}$.

the eye. Procedures adhered to the guidelines of the Association for Research in Vision and Ophthalmology Resolution on the Use of Animals in Research.

Rabbits were killed with an overdose of pentobarbitone sodium at 0 hours, at $1,3,5,7$, and 10 days, and at 2 weeks, 1,3 , and 6 months after the operation. At least four rabbits were studied at each specified time. Eyes were enucleated and immersed in $10 \%$ neutral buffered formalin. After fixation, the globe was sectioned at the equator. Specimens were dehydrated through a graded series of alcohols and embedded in paraffin. Sections were cut from specimens.

The labelled streptavidin-biotin method using a Histostein-SP kit (Zymed, South San Francisco, CA, USA) was used to detect

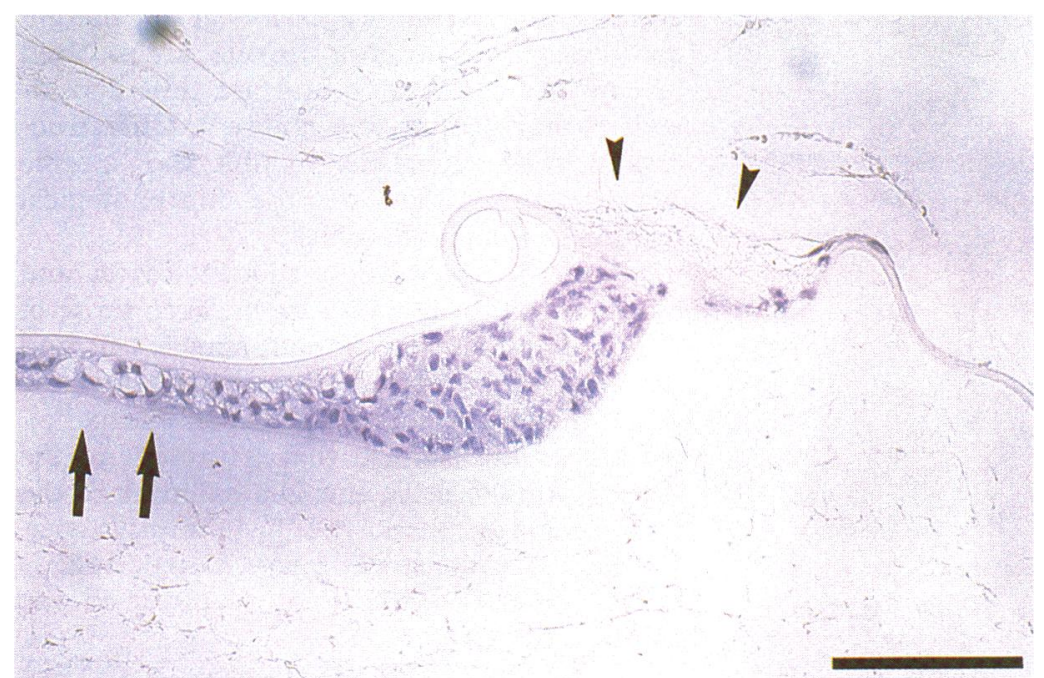

Figure 2 Immunolocalisation of $\alpha$ smooth muscle actin ( $\alpha-S M A)$ in aphakic rabbit eyes 3 days after cataract surgery. Monolayered and vacuolated lens epithelial cells (LECs) cover the inner surfaces of these capsules (arrows). LECs at the adhesive portion are multilayered and are surrounded by a fibrous material (arrowheads), but are not vacuolated. LECs are negative for $\alpha-S M A$. Sections were stained with

3-3'-diaminobenzidine hydrochloride and counterstained with haematoxylin. Bar $=100 \mu \mathrm{m}$.
$\alpha$-SMA immunohistochemically. Sections were deparaffinised with xylene, rehydrated in a graded series of alcohols, and immunostained for $\alpha$-SMA according to the manufacturer's instructions, with the following modifications. Endogenous peroxidase activity was quenched by treating the sections with $3 \%$ hydrogen peroxide in methanol for 20 minutes at room temperature. Non-specific background staining was eliminated by incubating the sections with non-immune goat serum (reagent $1 \mathrm{~A} ; \mathrm{H}-\mathrm{SP}$ kit) at room temperature. A primary mouse monoclonal antibody directed against $\alpha$-SMA (IgG2a, clone 1A4, code no M851, Dakopatts, Denmark) was diluted 1:100 with primary antibody diluting buffer (Biomedia Corp, Foster City, CA, USA). Sections were incubated with this diluted antibody in a moist chamber at room temperature for 30 minutes. Sections were then rinsed three times with phosphate buffered saline (PBS), incubated with a biotinylated secondary antibody (goat anti-mouse IgG; reagent $1 \mathrm{~B}$; $\mathrm{H}-\mathrm{SP}$ kit) at room temperature for 10 minutes, and washed three times with PBS. Streptavidin-peroxidase was added (reagent 2; H-SP kit), and the sections were incubated at room temperature for 15 minutes, then rinsed three times with PBS. Finally, peroxidase was visualised by the addition of a solution containing 3-3'diaminobenzidine hydrochloride $(0.3 \mathrm{mg} / \mathrm{ml})$, $0.005 \%$ hydrogen peroxide, and $50 \mathrm{mM}$ TRIS- $\mathrm{HCl}$ buffer at room temperature for 4 minutes. Sections were then counterstained with haematoxylin.

Mouse monoclonal IgG2a antibody (clone Dak-G05, code no X943, Dakopatts) was used as a negative control. No immunoreaction was detected in the negative control. The sphincter and dilator muscles of the iris were used as internal positive controls. ${ }^{22}$

\section{Results}

Some residual LECs were observed on the peripheral anterior capsule immediately after cataract surgery. Some cortical lens fibres also persisted. These LECs and lens fibres were negative for $\alpha$-SMA (data not shown).

On postoperative day 1 , the anterior capsule at the capsulotomy margin rolled up and adhered to the posterior capsule. This adhesion divided the postoperative lens into three portions: the central posterior capsule, the adhesive portion, and the capsular bag. The capsular bag consisted of the peripheral posterior capsule and anterior capsule. In the capsular bag, LECs on the anterior capsule began to flatten and migrate to the posterior capsule via the lens equator and the adhesive portion. However, LECs did not migrate to the central posterior capsule from the adhesive portion. LECs were negative for $\alpha$-SMA (Fig 1).

On postoperative day 3 , a monolayer of LECs covered the inner surface of the anterior and posterior capsules in the capsular bag. Some of these LECs were vacuolated. The capsules were attached to one another like a zipper. The LECs at the adhesive portion were multilayered but not vacuolated, and were sur- 


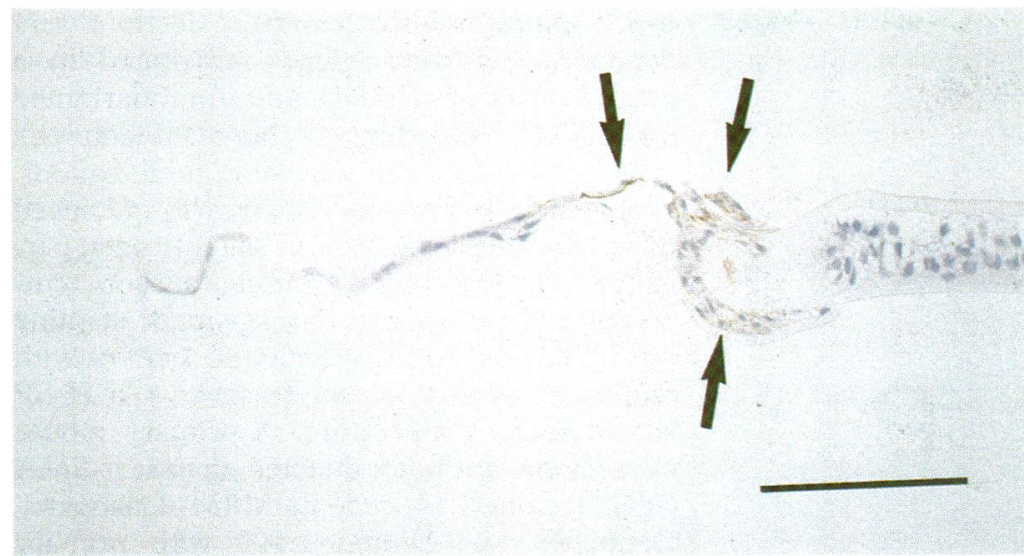

Figure 3 Immunolocalisation of $\alpha$ smooth muscle actin $(\alpha-S M A)$ in aphakic rabbit eyes 5 days after cataract surgery. Lens epithelial cells (LECs) at the adhesion portion had become flattened with spindle-shaped cross sections, and had begun to migrate to the central posterior capsule. These flattened LECs are positive for $\alpha-S M A$ (arrows) while the vacuolated LECs in capsular bag were negative for $\alpha-S M A$. Sections were stained with 3-3'-diaminobenzidine hydrochloride and counterstained with haematoxylin. Bar $=100 \mu \mathrm{m}$.

rounded by a fibrous material. Few LECs migrated to the central posterior capsule. These LECs were negative for $\alpha$-SMA (Fig 2).

On postoperative day 5 , the multilayered LECs at the adhesive portion were flattened with spindle-shaped cross sections, whereas the LECs in the capsular bag were monolayered and vacuolated. The fibrous material that had surrounded the LECs at the adhesive portion had disappeared and the LECs had begun to migrate to the central posterior capsule. Only the flattened LECs at the adhesive portion were positive for $\alpha$-SMA (Fig 3).
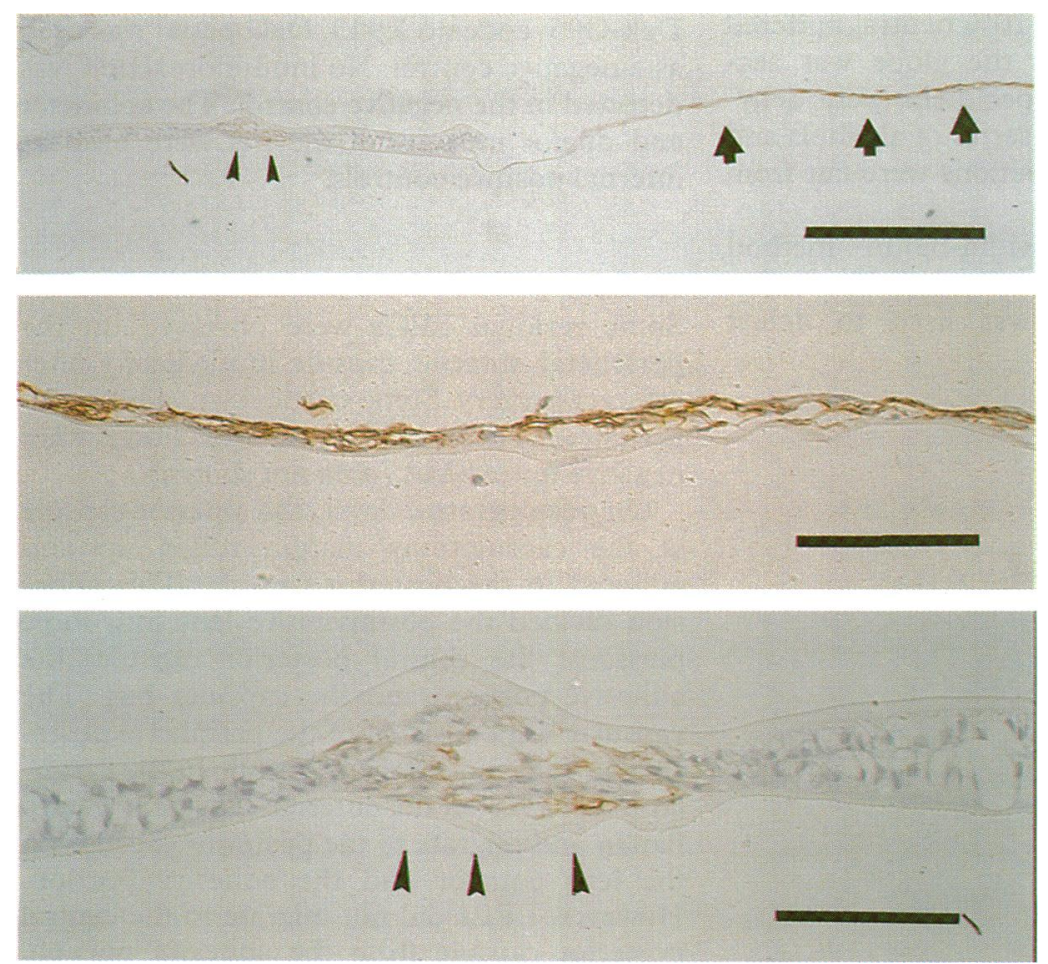

Figure 4 (Top) Immunolocalisation of $\alpha$ smooth muscle actin ( $\alpha-S M A)$ in aphakic rabbit eyes 7 days after cataract surgery. Flattened lens epithelial cells (LECs) that are $\alpha-S M A$ positive essentially cover the central posterior capsule (arrows). The capsular bag is occupied by vacuolated LECs and lens fibres that are negative for $\alpha-S M A$, but a clump of $\alpha-S M A$ positive flattened LECs is visible in the capsular bag (arrowheads). Bar $=500$ $\mu \mathrm{m}$. (Middle) Higher power view of posterior capsule covered with $\alpha-S M A$ positive, flattened LECs. Bar = $100 \mu \mathrm{m}$. (Bottom) Higher power view of clump of $\alpha-S M A$ positive flattened LECs in the capsular bag (arrowheads). The lens capsule around these LECs appears to be slightly wrinkled. Bar $=100 \mu \mathrm{m}$. Sections were stained with 3-3'-diaminobenzidine hydrochloride and counterstained with haematoxylin.
On postoperative day 7, the flattened LECs were $\alpha$-SMA positive, and covered almost the entire central posterior capsule (Fig 4 (top and middle)). The capsular bag was occupied by vacuolated LECs that were negative for $\alpha$-SMA. After the seventh postoperative day, clumps of $\alpha$-SMA positive, flattened LECs were occasionally visible in the capsular bag (Fig 4 (top and bottom)).

On postoperative day 10 , most of the flattened LECs that earlier had covered the central posterior capsule had now disappeared, whereas the flattened LECs at the adhesive portion remained. The remaining flattened LECs were positive for $\alpha$-SMA (Fig 5).

After postoperative day 14, the LECs that covered the inner surface of the anterior capsule exhibited a normal cuboidal appearance. The LECs gradually became differentiated. The capsular bag was now filled with fibres and had formed Soemmerring's ring. However, the LECs at the adhesive portion were flattened and positive for $\alpha$-SMA. The posterior capsule was wrinkled at the adhesive portion, to which the $\alpha$-SMA positive LECs were attached (Fig 6). At this time, the LECs did not migrate to the central posterior capsule.

\section{Discussion}

After the fifth postoperative day, flattened LECs were observed mainly around the adhesive portion of the anterior capsule margin and the central posterior capsule, and were present only transiently on the central posterior capsule. These flattened LECs could be distinguished morphologically from the monolayered LECs in the capsular bag, which showed early vacuolation and later became cuboidal. The flattened LECs related to the wrinkling of the lens capsule. This behaviour of the flattened LECs resembled that described previously by other investigators. ${ }^{4-6}$ However, the present study also demonstrated that these flattened LECs were $\alpha$-SMA positive.

Flattened LECs, especially around the adhesive portion of the anterior capsule margin and the central posterior capsule, are involved in capsular contraction and are thus considered to have contractile ability. ${ }^{4-7}$ Ultrastructurally, they reportedly exhibit the specific microfilaments that are the morphological features of the myofibroblasts. ${ }^{45}$

Myofibroblasts possess morphological and biochemical features that are between those of the fibroblast and the smooth muscle cell, and are involved in retractile phenomena and the accumulation of the extracellular matrix. ${ }^{13}{ }^{14}$ $\alpha$-SMA is the isoform that is present in the smooth muscle cells, and is a hallmark of the myofibroblastic phenotype. ${ }^{1314}$ Our results suggested that 5 days after surgery, flattened LECs possessed the biochemical features of the myofibroblast.

Five days after cataract surgery, the $\alpha$-SMA positive LECs were observed mainly around the adhesive portion of the anterior capsule margin and the posterior capsule, and were present only transiently on the central posterior capsule. Active transforming growth factor 


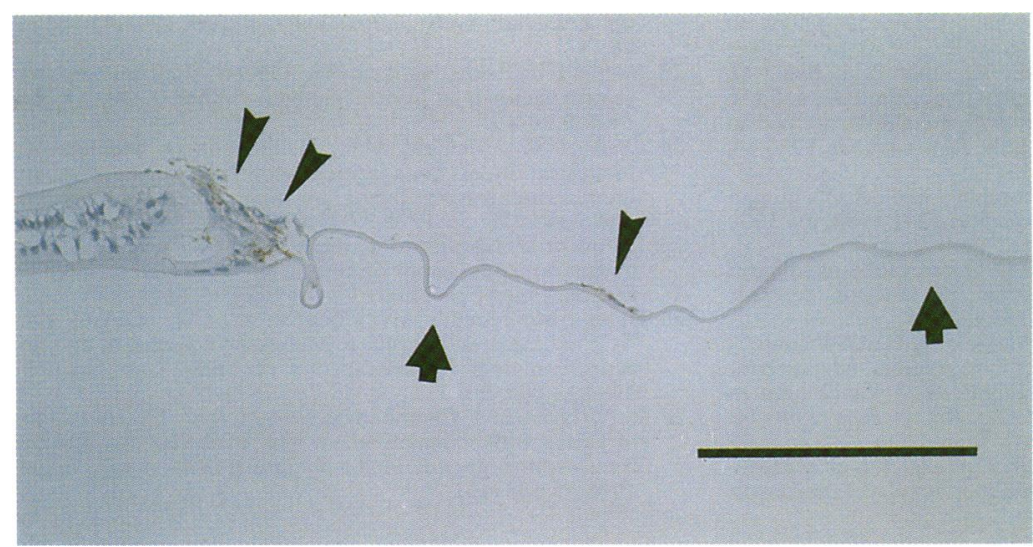

Figure 5 Immunolocalisation of $\alpha$ smooth muscle actin ( $\alpha-S M A)$ in aphakic rabbit eyes 10 days after cataract surgery. $A$ few flattened lens epithelial cells (LECs) are present at the central posterior capsule (arrows). Flattened LECs at the adhesive portion and central posterior capsule are $\alpha-S M A$ positive (arrowheads). Sections were stained with 3-3'-diaminobenzidine hydrochloride and counterstained with haematoxylin. Bar $=100 \mu \mathrm{m}$.

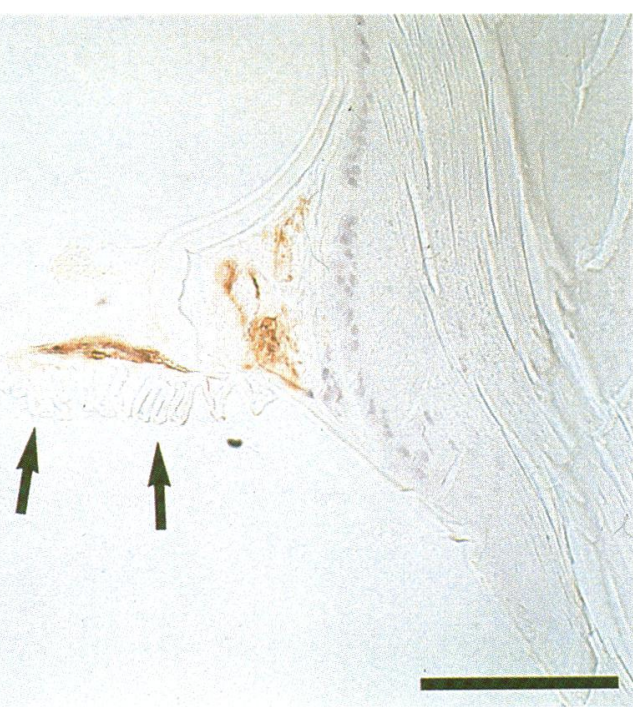

Figure 6 Immunolocalisation of $\alpha$ smooth muscle actin ( $\alpha-S M A)$ in aphakic rabbit eyes 6 months after cataract surgery. The capsular bag is filled with lens fibres and has formed Soemmerring's ring. Flattened lens epithelial cells that are $\alpha-S M A$ positive are present at the adhesive portion. The posterior capsule is wrinkled at this adhesive portion (arrows). Sections were stained with 3-3'-diaminobenzidine hydrochloride and counterstained with haematoxylin. Bar $=100 \mu \mathrm{m}$.

B2 (TGF-B2) is present in the aqueous humour of humans and rabbits. ${ }^{23-25}$ It was recently found that TGF- $B$ s increase the expression of $\alpha$-SMA in cultured LECs. ${ }^{17}{ }^{26}$ These observations suggest that the TGF- $B 2$ in the aqueous humour may play a role in the presence of $\alpha$-SMA in LECs.

In this study, the $\alpha$-SMA positive LECs disappeared from the central posterior capsule 10 days after the operation although they had almost covered the central posterior capsule 3 days earlier. They were not observed thereafter. In aphakic dog eyes, LECs are present in the central posterior capsule 7 days after surgery, but are not observed 10 weeks after surgery. ${ }^{6} \mathrm{Liu} e \mathrm{el}^{27}$ reported that the majority of rat LECs disappear from explants that were exposed to TGF- $B$ for 5 days. These observations suggest that TGF- $\beta$ may also be involved in the disappearance of LECs from the central posterior capsule. However, in aphakic human eyes, LECs have been found in the central posterior capsule as long as 2 years postoperatively. ${ }^{3}$ The reason for these species differences is not known.
The present study showed the presence of $\alpha$-SMA in the LECs but not in the intercellular stroma. However, Frezzotti et al ${ }^{21}$ described the presence of $\alpha-S M A$ filaments in the intercellular stroma, but not in the LECs, of the fibrotic membrane that was removed surgically from the surface of the optic of the intraocular lens of the patient. In a study of monkey eyes, most LECs around the anterior capsule margin showed signs of degeneration 12 months after cataract surgery; ultrastructurally, cellular debris was observed around these cells. ${ }^{7}$ We suspect that some of the cellular debris in the stroma may have shown positivity for $\alpha$-SMA, although Frezzotti et al ${ }^{21}$ did not specify the period following cataract surgery at which the membrane had been removed.

In conclusion, the present study showed that the flattened LECs seen 5 days after cataract surgery and that are related to a wrinkling of the posterior capsule were positive for $\alpha$-SMA. Cuboidal LECs were negative for $\alpha$-SMA. Thus, one can distinguish the flattened LECs from the normal LECs biochemically by the presence of $\alpha$-SMA.

We thank Professor Y Oguchi for his valuable suggestions on the manuscript.

manuscript.
This work was supported in part by a grant in aid (No This work was supported in part by a grant in aid (No
08771527 ) for scientific research from the Ministry of Education, Science, and Culture of Japan.

1 Blomstedt G, Fargerholm P, Gallo J, Philipson B. Aftercataract in the rabbit eye following extracapsular cataract extraction-a wound healing reaction. Acta Ophthalmol 1987;65 (suppl 182):93-9.

2 Gwon AE, Gruber LJ, Mundwiler KE. A histologic study of lens regeneration in aphakic rabbits. Invest Ophthalmol Vis Sci 1990;31:540-7.

3 McDonnell PJ, Stark WJ, Green R. Posterior capsule opacification: a specular microscopic study. Ophthalmology 1984;91:853-6.

4 McDonnell PJ, Zarbin MA, Green WR. Posterior capsule opacification in pseudophakic eyes. Ophthalmology 1983; 90:1548-53.

5 Cobo LM, Ohsawa E, Chandler D, Arguello R, George G. Pathogenesis of capsular opacification after extracapsular cataract extraction: an animal model. Ophthalmology 1984; 91:857-63.

6 Nasisse MP, Dykstra MJ, Cobo LM. Lens capsule opacification in aphakic and pseudophakic eyes. Graefes opacification in aphakic and pseudophakic

7 Ishibashi T, Araki H, Sugai S, Tawara A, Ohnishi Y, Inomata $\mathrm{H}$. Anterior capsule opacification in monkey eyes with posterior chamber intraocular lenses. Arch Ophthalmol 1993; 111:1685-90.

8 Davison JA. Capsule contraction syndrome. $f$ Cataract Refract Surg 1993;19:582-9.

9 Hansen SO, Crandall AS, Olson RJ. Progressive constriction of the anterior capsular opening following intact capsulorhexis. $\mathcal{F}$ Cataract Refract Surg 1993;19:77-82.

10 Masket S. Postoperative complications of capsulorhexis. $f$ Cataract Refract Surg 1993;19:721-4.

11 Katsura H, Oda H, Utsumi Y. Breaks in the pars plicata following surgery for atopic cataract. Ophthalmic Surg 1994;25:514-5.

12 Volkmann U, Kampik A. Late hypotony after posterio chamber lens implantation. Klin Monatsbl Augenheilkd 1990;197:418-21.

13 Sappino AP, Schurch W, Gabbiani G. Biology of disease: differentiation repertoire of fibroblastic cells: expression of cytoskeletal proteins as marker of phenotypic modulations. cytoskeletal proteins as mark
Lab Invest 1990;63:144-61.

14 Desmoulière A, Geinoz A, Gabbiani F, Gabbiani G. Transforming growth factor- $\beta 1$ induces $\alpha$-smooth muscle actin expression in granulation tissue myofibroblasts and quiescent and growing cultured fibroblasts. $\mathcal{F}$ Cell Biol 1993;122: 103-11.

15 Arora PD, McCulloch CA. Dependence of collagen remodelling on alpha-smooth muscle actin expression by fibroblasts. f Cell Physiol 1994;159:161-75.

16 Grisanti S, Guidry C. Transdifferentiation of retinal pigment epithelial cells from epithelial to mesenchyma phenotype. Invest Ophthalmol Vis Sci 1995;36:361-405.

17 Kurosaka D, Kato K, Nagamoto T, Negishi K. Growth factors influence contractility and $\alpha$-smooth muscle actin 
expression in bovine lens epithelial cells. Invest Ophthalmol $V$ is $S$ ci 1995;36:700-8.

18 Schmitt-Gräff A, Pau H, Spahr R, Piper HM, Skalli O, Gabbiani G. Appearance of alpha-smooth muscle actin in human eye lens cells of anterior capsular cataract and in cultured bovine lens-forming cells. Differentiation 1990;43: 115-22.

19 Novotony GEK, Pau H. Myofibroblast-like cells in human anterior capsular cataract. Virchows Arch A 1984;404:393 401 .

20 Novotony GEK, Pau H, Arnold G. Organization of collagen and other extracellular material in anterior capsular cataract. Anat Anz fena 1989;168:127-33.

21 Frezzotti R, Caporossi A, Mastrangelo D, Hadjistilianou T, Tosi $\mathrm{P}$, Cintorino $\mathrm{M}$, et al. Pathogenesis of posterior capsu-lar opacification. Part II: histopathological and in vitro culture findings. F Cataract Refract Surg 1990;16: 353-60.

22 Kivelä T, Fuchs U, Tarkkanen A. Cytoskeleton in neuroectodermally derived epithelial and muscle cells of the human iris and ciliary body. $\mathcal{f}$ Histochem Cytochem 1992;40:1517-

23 Jampel HD, Roche N, Stark WJ, Roberts AB. Transforming growth factor- $B$ in human aqueous humor. Curr Eye Res 1990;9:963-9.

24 Cousins SW, McCabe MM, Danielpour D, Streilein W. Identification of transforming growth factor-beta as an immunosuppressive factor in aqueous humor. Invest Ophthalmol Vis Sci 1991;32:2201-11.

25 Kurosaka D, Nagamoto T. Inhibitory effect of TGF-B2 in human aqueous humor on bovine lens epithelial cell prolifhuman aqueous humor on bovine lens epithelial cell

26 Hales AM, Schultz MW, Chamberlain CG, McAvoy JW. TGF- $\beta 1$ induces lens cells to accumulate $\alpha$-smooth muscle actin, a marker for subcapsular cataracts. Curr Eye Res 1994;13:885-90.

27 Liu J, Hales AM, Chamberlain CG, McAvoy JW. Induction of cataract-like changes in rat lens epithelial explants by transforming growth factor B. Invest Ophthalmol Vis $\mathrm{Sci}$ 1994;35:388-401. 